\title{
Study of some system of nonlinear partial differential equations by LDM and MLDM
}

\author{
S. S. Handibag' 1 , R. M. Wayal ${ }^{2}$ \\ ${ }^{1}$ Department of Mathematics, Mahatma Basweshar Mahavidyalaya, Latur-413 512, \\ Maharashtra, India, Email Id: sujitmaths@ gmail.com \\ ${ }^{2}$ Department of Mathematics, Hutatma Rajguru Mahavidyalaya, Rajgurunagar-410 505, \\ Maharashtra, India, Email Id: rupalimaths27@gmail.com \\ DOI: 10.29322/IJSRP.11.06.2021.p11459 \\ http://dx.doi.org/10.29322/IJSRP.11.06.2021.p11459
}

\begin{abstract}
:
In the present article substantial Mathematical technique namely LDM and modified LDM has been employed to find the analytical solution of system of NLPDEs. Reliability of these methods were examined by illustrating three examples viz. Drinfield Sokolov (DS) system, coupled Burger's equation and Cauchy problem. Both the methods have many advantages, they converge expeditiously to the exact solution also they do not require linearization, discretization or perturbation. Result obtained by these methods affirms that proposed methods are easy, powerful and efficient technique to find solution of wide class of system on NLPDs.
\end{abstract}

Key words: LDM, MLDM, Adomian polynomials and system of NLPDEs.

\section{Introduction:}

Every scientific field is ubiquitous by NLPDEs for an instant control system, climate change, plasma physics, elastic media, oceanology, meteorology, mathematical biology, geophysics, nonlinear optics, chemical reaction, gravitation, fluid mechanics, solid state physics and others. Hence to comprehend the behavior of different physical phenomena's solution of NLPDEs plays crucial role. To find the solution of system of NLPDEs different techniques are available such as First integral method [1], ADM [2], Exp-function [3], Tanh function method [4], Tanh-Coth [5], HPM [6], HBM [7], LDM [8], MLDM [9], Differential transformation methods [10], Jacobi's elliptic function method [11] etc.

The main focus of this paper is to present well grounded technique to find the solution of system of nonlinear partial differential equations called LDM which was introduced by Khuri, for finding the solution of class of NLPDEs in 2001 [12]. Hussain and Khan modified this technique in 2010 [9]. Solution of coupled Burger's equation and Cauchy problem are obtained by LDM and Drinfield Sokolov system solved by MLDM. Coupled Burger's equation was derived by Esipov to describe the model of polydispersive sedimentation in 1995[13]. Approximation theory of flow through a shock wave traveling in a viscous fluid is described with the help of coupled Burger's equation [14]. It has crucial role in different physical phenomena viz. shock wave, hydrodynamic turbulence, dispersion in porous media and vorticity transport etc. DS equation represents nonlinear diffusion equation and possesses Lax pairs of a special form. Wazwaz found travelling wave solutions with compact and noncompact structures of DS system by using sine-cosine and tanh method [15]. F. Zhang, J.Qi and W. Yuan employed complex method to derive general meromorphic solutions of DS system [16]. A bifurcation phenomenon of nonlinear waves for DS system was studied by H. Cai, C. Pan and Z. Liu [17]. Jing Wang gave different properties of DS system like Hamiltonian, symplectic, cosymplectic, recursion operator, scaling symmetry and roots of symmetries [18]. Cnoidal, snoidal, periodic, solitary and compacton wave solutions of DS system were obtained by M. Inc, E. Fendoglu, H. Triki and A. Biswas by using Jacobi's elliptic function method [11].

This paper is divided into 4 sessions, in session 2 Introduction of LDM and MLDM is given, applications given in session 3. Paper is concluded in session 4.

\section{Laplace Decomposition Method:[12]}

Consider the system of NLPD equations

$$
\mathcal{L} \mathrm{u}_{\mathrm{i}}(\mathrm{x}, \mathrm{t})+\mathcal{R} \mathrm{u}_{\mathrm{i}}(\mathrm{x}, \mathrm{t})+\mathcal{N} \mathrm{u}_{\mathrm{i}}(\mathrm{x}, \mathrm{t})=0, \quad \mathrm{i}=1,2, \ldots, \mathrm{n}
$$

With initial condition 


$$
\mathrm{u}_{\mathrm{i}}(\mathrm{x}, 0)=\mathrm{f}_{\mathrm{i}}(\mathrm{x})
$$

where $\mathcal{L}=\frac{\partial}{\partial \mathrm{t}}, \mathcal{R}$ is general linear operator, $\mathcal{N} u$ is nonlinear term. Taking Laplace transform of (1) w.r.t. $t$

$$
\begin{gathered}
\mathrm{su}_{\mathrm{i}}(\mathrm{x}, \mathrm{s})-\mathrm{u}_{\mathrm{i}}(\mathrm{x}, 0)=-\mathfrak{L}_{\mathrm{t}}\left[\mathcal{R} \mathrm{u}_{\mathrm{i}}(\mathrm{x}, \mathrm{t})+\mathcal{N} \mathrm{u}_{\mathrm{i}}(\mathrm{x}, \mathrm{t})\right] \\
\mathrm{u}_{\mathrm{i}}(\mathrm{x}, \mathrm{s})=\frac{\mathrm{f}_{\mathrm{i}}(\mathrm{x})}{\mathrm{s}}-\frac{1}{\mathrm{~s}} \mathfrak{Q}_{\mathrm{t}}\left[\mathcal{R} \mathrm{u}_{\mathrm{i}}(\mathrm{x}, \mathrm{t})+\mathcal{N} \mathrm{u}_{\mathrm{i}}(\mathrm{x}, \mathrm{t})\right]
\end{gathered}
$$

Taking inverse Laplace transform

$$
\mathrm{u}_{\mathrm{i}}(\mathrm{x}, \mathrm{t})=\mathrm{f}_{\mathrm{i}}(\mathrm{x})-\mathfrak{Q}_{\mathrm{t}}^{-1}\left\{\frac{1}{\mathrm{~s}} \mathfrak{L}_{\mathrm{t}}\left[\mathcal{R} \mathrm{u}_{\mathrm{i}}(\mathrm{x}, \mathrm{t})+\mathcal{N} \mathrm{u}_{\mathrm{i}}(\mathrm{x}, \mathrm{t})\right]\right\}
$$

Let solution of the equation (1) is in series form

$$
\mathrm{u}_{\mathrm{i}}(\mathrm{x}, \mathrm{t})=\sum_{\mathrm{n}=0}^{\infty} \mathrm{u}_{\mathrm{i} n}(\mathrm{x}, \mathrm{t})
$$

Nonlinear term can be decomposed with the help of Adomian polynomials.

$$
\mathcal{N} \mathrm{u}_{\mathrm{i}}=\sum_{\mathrm{n}=0}^{\infty} \mathcal{A}_{\mathrm{i}_{\mathrm{n}}}
$$

Where $\mathcal{A}_{i_{n}}{ }^{\prime} s$ are Adomian polynomials which can be calculated by using following relation [19]

$$
\mathcal{A}_{\mathrm{i}_{\mathrm{n}}}=\frac{1}{\mathrm{n} !} \frac{\mathrm{d}^{\mathrm{n}}}{\mathrm{d} \lambda^{\mathrm{n}}}\left[\mathcal{N}\left(\sum_{\mathrm{r}=0}^{\infty} \lambda^{\mathrm{r}} \mathrm{u}_{\mathrm{i}_{\mathrm{r}}}\right)\right]_{\lambda=0}, \mathrm{n} \geq 0
$$

From equation (2) to (4)

$$
\sum_{\mathrm{n}=0}^{\infty} \mathrm{u}_{\mathrm{i}_{\mathrm{n}}}(\mathrm{x}, \mathrm{t})=\mathrm{f}_{\mathrm{i}}(\mathrm{x})-\mathfrak{R}_{\mathrm{t}}^{-1}\left\{\frac{1}{\mathrm{~s}} \mathfrak{L}_{\mathrm{t}}\left[\mathcal{R} \sum_{\mathrm{n}=0}^{\infty} \mathrm{u}_{\mathrm{i}_{\mathrm{n}}}(\mathrm{x}, \mathrm{t})+\sum_{\mathrm{n}=0}^{\infty} \mathcal{A}_{\mathrm{i}_{\mathrm{n}}}\right]\right\}
$$

Where

$$
\begin{gathered}
\mathrm{u}_{\mathrm{i}_{0}}(\mathrm{x}, \mathrm{t})=\mathrm{f}_{\mathrm{i}}(\mathrm{x}) \\
\mathrm{u}_{\mathrm{i}_{\mathrm{n}+1}}(\mathrm{x}, \mathrm{t})=-\mathfrak{Q}_{\mathrm{t}}^{-1}\left\{\frac{1}{\mathrm{~s}} \mathfrak{Q}_{\mathrm{t}}\left[\mathcal{R}\left(\mathrm{u}_{\mathrm{i}_{\mathrm{n}}}\right)+\mathcal{A}_{\mathrm{i}_{\mathrm{n}}}\right]\right\}
\end{gathered}
$$

Where $\mathrm{n}=0,1,2,3, \ldots$

Then series solution of equation (1) is

$$
u_{i}(x, t)=u_{i_{0}}+u_{i_{1}}+u_{i_{2}}+u_{i_{3}}+\cdots \quad, \quad i=1,2,3, \ldots
$$

In modified Laplace decomposition method there is only slide change

If

$$
\mathrm{f}_{\mathrm{i}}(\mathrm{x})=\mathrm{f}_{\mathrm{i} 1}+\mathrm{f}_{\mathrm{i} 2}
$$

Then take

$$
\mathrm{u}_{\mathrm{i}_{0}}(\mathrm{x}, \mathrm{t})=\mathrm{f}_{\mathrm{i} 1}(\mathrm{x})
$$

$$
\mathrm{u}_{\mathrm{i}_{1}}(\mathrm{x}, \mathrm{t})=\mathrm{f}_{\mathrm{i} 2}-\mathfrak{R}_{\mathrm{t}}^{-1}\left\{\frac{1}{\mathrm{~s}} \mathfrak{R}_{\mathrm{t}}\left[\mathcal{R}\left(\mathrm{u}_{\mathrm{i}_{0}}\right)+\mathcal{A}_{\mathrm{i}_{0}}\right]\right\}
$$

And

$$
\mathrm{u}_{\mathrm{i}_{\mathrm{n}+1}}(\mathrm{x}, \mathrm{t})=-\mathfrak{Q}_{\mathrm{t}}^{-1}\left\{\frac{1}{\mathrm{~s}} \mathfrak{L}_{\mathrm{t}}\left[\mathcal{R}\left(\mathrm{u}_{\mathrm{i}_{\mathrm{n}}}\right)+\mathcal{A}_{\mathrm{i}_{\mathrm{n}}}\right]\right\}, \quad \text { where } n=1,2,3, \ldots
$$

\section{Applications:}

Example 1: Consider coupled Burger's equation [20]

$$
\begin{aligned}
& u_{t}-u_{x x}-2 u u_{x}+(u v)_{x}=0 \\
& v_{t}-v_{x x}-2 v v_{x}+(u v)_{x}=0
\end{aligned}
$$

With initial condition $\quad u(x, 0)=\sin x, \quad v(x, 0)=\sin x$

Taking Laplace transform w.r.t. $\mathrm{t}$

$$
s u(x, s)-u(x, 0)=\mathfrak{Q}_{t}\left[u_{x x}+2 u u_{x}-(u v)_{x}\right]
$$




$$
\begin{aligned}
& s v(x, s)-v(x, 0)=\mathfrak{L}_{t}\left[v_{x x}+2 v v_{x}-(u v)_{x}\right] \\
& u(x, s)=\frac{u(x, 0)}{s}+\frac{1}{s} \mathfrak{L}_{t}\left[u_{x x}+2 u u_{x}-(u v)_{x}\right] \\
& v(x, s)=\frac{v(x, 0)}{s}+\frac{1}{s} \mathfrak{L}_{t}\left[v_{x x}+2 v v_{x}-(u v)_{x}\right] \\
& u(x, s)=\frac{\sin x}{s}+\frac{1}{s} \mathfrak{L}_{t}\left[u_{x x}+2 u u_{x}-(u v)_{x}\right] \\
& v(x, s)=\frac{\sin x}{s}+\frac{1}{s} \mathfrak{L}_{t}\left[v_{x x}+2 v v_{x}-(u v)_{x}\right]
\end{aligned}
$$

By taking inverse Laplace transform

$$
\begin{aligned}
& u(x, t)=\sin x+\mathfrak{L}_{t}^{-1}\left\{\frac{1}{s} \mathfrak{L}_{t}\left[u_{x x}+2 u u_{x}-(u v)_{x}\right]\right\} \\
& v(x, t)=\sin x+\mathfrak{L}_{t}^{-1}\left\{\frac{1}{s} \mathfrak{L}_{t}\left[v_{x x}+2 v v_{x}-(u v)_{x}\right]\right\}
\end{aligned}
$$

Let $\quad u(x, t)=\sum_{n=0}^{\infty} u_{n}(x, t)$ and $\quad v(x, t)=\sum_{n=0}^{\infty} v_{n}(x, t)$ be solutions of equation (7)

$$
\begin{gathered}
u u_{x}=\sum_{n=0}^{\infty} \mathcal{A}_{n}, \quad v v_{x}=\sum_{n=0}^{\infty} \mathcal{B}_{n} \text { and }(u v)_{x}=\sum_{n=0}^{\infty} \mathcal{C}_{n} \\
\therefore \sum_{n=0}^{\infty} u_{n}(x, t)=\sin x+\mathfrak{L}_{t}^{-1}\left\{\frac{1}{s} \mathfrak{L}_{t}\left[\sum_{n=0}^{\infty} u_{n_{x x}}+2 \sum_{n=0}^{\infty} \mathcal{A}_{n}-\sum_{n=0}^{\infty} \mathcal{C}_{n}\right]\right\} \\
\therefore \sum_{n=0}^{\infty} v_{n}(x, t)=\sin x+\mathfrak{L}_{t}^{-1}\left\{\frac{1}{s} \mathfrak{L}_{t}\left[\sum_{n=0}^{\infty} v_{n_{x x}}+2 \sum_{n=0}^{\infty} \mathcal{B}_{n}-\sum_{n=0}^{\infty} \mathcal{C}_{n}\right]\right\}
\end{gathered}
$$

Consider

$$
\begin{aligned}
& u_{0}(x, t)=\sin x \quad \text { and } \quad v_{0}(x, t)=\sin x \\
& u_{n+1}(x, t)=\mathfrak{L}_{t}^{-1}\left\{\frac{1}{s} \mathfrak{L}_{t}\left[u_{n_{x x}}+2 \mathcal{A}_{n}-\mathcal{C}_{n}\right]\right\} \\
& v_{n+1}(x, t)=\mathfrak{L}_{t}^{-1}\left\{\frac{1}{s} \mathfrak{\Omega}_{t}\left[v_{n_{x x}}+2 \mathcal{B}_{n}-\mathcal{C}_{n}\right]\right\}
\end{aligned}
$$

Some Adomian polynomials are

$$
\begin{array}{ll}
\mathcal{A}_{0}=u_{0} u_{0 x} & \mathcal{B}_{0}=v_{0} v_{0 x} \\
\mathcal{A}_{1}=u_{0} u_{1 x}+u_{1} u_{0 x} & \mathcal{B}_{1}=v_{0} v_{1 x}+v_{1} v_{0 x} \\
\mathcal{A}_{2}=u_{0} u_{2 x}+u_{1} u_{1 x}+u_{2} u_{0 x} & \mathcal{B}_{1}=v_{0} v_{2 x}+v_{1} v_{1 x}+v_{2} v_{0 x} \\
\mathcal{C}_{0}=u_{0} v_{0 x}+u_{0 x} v_{0} & \\
\mathcal{C}_{1}=u_{0} v_{1 x}+u_{1} v_{0 x}+u_{1 x} v_{0}+u_{0 x} v_{1} & \\
\mathcal{C}_{2}=u_{0} v_{2 x}+u_{1} v_{1 x}+u_{2} v_{0 x}+u_{2 x} v_{0}+u_{0 x} v_{2}+u_{1 x} v_{1}
\end{array}
$$

From equation (8) and (9) we get

$$
\begin{array}{cc}
u_{1}(x, t)=-\sin x t, & v_{1}(x, t)=-\sin x t \\
u_{2}(x, t)=\sin x \frac{t^{2}}{2 !}, & v_{2}(x, t)=\sin x \frac{t^{2}}{2 !} \\
u_{3}(x, t)=-\sin x \frac{t^{3}}{3 !}, & v_{2}(x, t)=-\sin x \frac{t^{3}}{3 !}
\end{array}
$$




$$
u_{4}(x, t)=\sin x \frac{t^{4}}{4 !}, \quad v_{4}(x, t)=\sin x \frac{t^{4}}{4 !} \ldots \ldots \text { so on }
$$

Hence series solution of equation (7) with given initial conditions is

$$
\begin{gathered}
u(x, t)=-\sin x t+\sin x \frac{t^{2}}{2 !}-\sin x \frac{t^{3}}{3 !}+\sin x \frac{t^{4}}{4 !}-\cdots \\
u(x, y)=\sin x\left[-t+\frac{t^{2}}{2 !}-\frac{t^{3}}{3 !}+\frac{t^{4}}{4 !}-\frac{t^{5}}{5 !}+\cdots\right] \\
v(x, y)=\sin x\left[-t+\frac{t^{2}}{2 !}-\frac{t^{3}}{3 !}+\frac{t^{4}}{4 !}-\frac{t^{5}}{5 !}+\cdots\right]
\end{gathered}
$$

These series converge to

$$
u(x, t)=e^{-t} \sin x \text { and } v(x, t)=e^{-t} \sin x
$$

This is exact solution of equation (7). Result is verified by substitution.

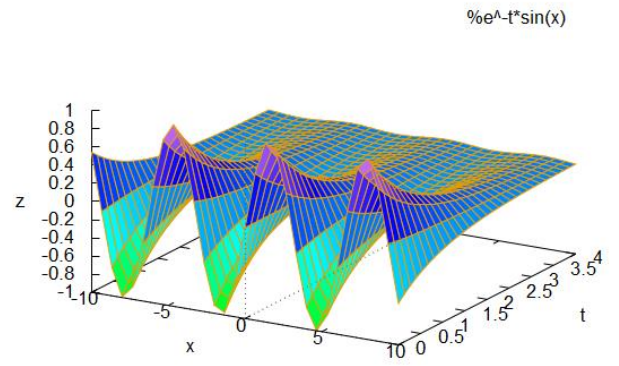

(a) $u(x, t)=e^{-t} \sin x$

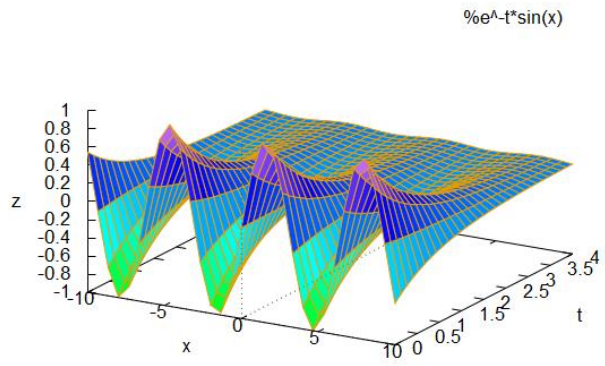

(b) $\mathbf{v}(\mathbf{x}, \mathbf{t})=\mathbf{e}^{-\mathbf{t}} \sin \mathbf{x}$

Figure 1: Graph of solution of coupled Burger's equation (a) $\boldsymbol{u}(\boldsymbol{x}, \boldsymbol{t})=\boldsymbol{e}^{-\boldsymbol{t}} \sin \boldsymbol{x}$, (b) $\boldsymbol{v}(\boldsymbol{x}, \boldsymbol{t})=\boldsymbol{e}^{-t} \sin \boldsymbol{x}$ for $-\mathbf{1 0} \leq \boldsymbol{x} \leq$ 10 and $0 \leq t \leq 4$.

Example 2: Consider Cauchy problem [21]

$$
\begin{aligned}
& u_{t}+\left(\frac{1}{2}\left(\frac{1}{2} u^{2}+v^{2}\right)+v\right)_{x}=0 \\
& v_{t}+(u v)_{x}=0
\end{aligned}
$$

With initial condition

$$
u(x, 0)=\frac{2 x}{10} \quad \text { and } \quad v(x, 0)=-\frac{110}{100}
$$

In this case we get following relation

$$
\begin{aligned}
& u_{0}(x, t)=\frac{2 x}{10}, \quad \text { and } v_{0}(x, t)=-\frac{110}{100} \\
& u_{n+1}=-\mathfrak{L}_{t}^{-1}\left\{\frac{1}{s} \mathfrak{L}_{t}\left[\frac{1}{4} \mathcal{D}_{n}+\frac{1}{2} \mathcal{E}_{n}\right]+v_{n x}\right\} \\
& v_{n+1}(x, t)=-\mathfrak{L}_{t}^{-1}\left\{\frac{1}{s} \mathfrak{L}_{t}\left[\mathcal{C}_{n}\right]\right\}
\end{aligned}
$$

Where $\mathcal{D}_{n}$ and $\mathcal{E}_{n}$ are Adomian polynomials, first few are as follows

$$
\begin{array}{ll}
\mathcal{D}_{0}=2 u_{0} u_{0 x} & \mathcal{E}_{0}=2 v_{0} v_{0 x} \\
\mathcal{D}_{1}=2 u_{1} u_{0 x}+2 u_{0} u_{1 x} & \mathcal{E}_{0}=2 v_{1} v_{0 x}+2 v_{0} v_{1 x} \\
\mathcal{D}_{2}=2 u_{1} u_{0 x}+2 u_{0} u_{1 x} & \mathcal{E}_{0}=2 v_{1} v_{0 x}+2 v_{0} v_{1 x}
\end{array}
$$

By using equation (16) and (17) first few components of the series are 

ISSN 2250-3153

$$
\begin{array}{cr}
u_{1}(x, t)=-2 x\left(\frac{t}{10^{2}}\right), & v_{1}(x, t)=110\left(\frac{2 t}{10^{3}}\right) \\
u_{2}(x, t)=2 x\left(\frac{t^{2}}{10^{3}}\right), & v_{2}(x, t)=-110\left(\frac{3 t^{2}}{10^{4}}\right) \\
u_{3}(x, t)=-2 x\left(\frac{t^{3}}{10^{4}}\right), & v_{3}(x, t)=110\left(\frac{4 t^{3}}{10^{5}}\right) \ldots \text { so on }
\end{array}
$$

Series solution of equation (14) with initial condition (15) is

$$
\begin{gathered}
u(x, t)=\frac{2 x}{10}-2 x\left(\frac{t}{10^{2}}\right)+2 x\left(\frac{t^{2}}{10^{3}}\right)-2 x\left(\frac{t^{3}}{10^{4}}\right)+\cdots \\
u(x, t)=\frac{2 x}{10}\left[1-\frac{t}{10}+\left(\frac{t}{10}\right)^{2}-\left(\frac{t}{10}\right)^{3}+\left(\frac{t}{10}\right)^{4}-\cdots\right] \\
v(x, t)=-\frac{110}{100}+\frac{110}{100}\left(\frac{2 t}{10}\right)-\frac{110}{100}\left(\frac{3 t^{2}}{10^{2}}\right)+\frac{110}{100}\left(\frac{4 t^{3}}{10^{3}}\right)-\cdots
\end{gathered}
$$

Above series converges to

$$
\begin{aligned}
& u(x, t)=\frac{2 x}{10+t}, \quad \quad \text { provided } t \neq-10 \\
& v(x, t)=-\frac{110}{(10+t)^{2}}, \quad \text { provided } \quad t \neq-10
\end{aligned}
$$

Which is exact solution of equation (14).
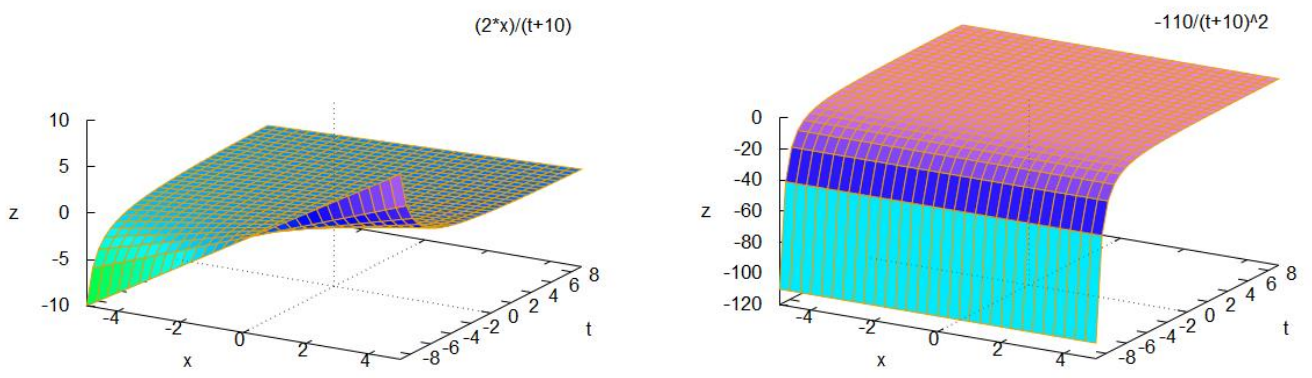
(a) $u(x, t)=\frac{2 x}{10+t}$
(b) $v(x, t)=\frac{-110}{(10+t)^{2}}$

Figure 2: Graph of solution of Cauchy problem for $-5 \leq x \leq 5$ and $-9 \leq t \leq 9$.

Example 3: Consider Drinfield Sokolov system [22]

$$
\begin{aligned}
& u_{t}+\left(v^{2}\right)_{x}=1-2(t-x) \\
& v_{t}-v_{x x x}+(u v)_{x}=1-2 x
\end{aligned}
$$

With initial condition $u(x, 0)=x$ and $v(x, 0)=-x$

In this case we used modified LDM

Taking Laplace transform

$$
\begin{gathered}
s u(x, s)-u(x, 0)=\frac{1}{s}-\frac{-2}{s^{2}}+\frac{2 x}{s}-\mathfrak{L}_{t}\left[\left(v^{2}\right)_{x}\right] \\
s v(x, s)-v(x, 0)=\frac{1}{s}-\frac{2 x}{s}+\mathfrak{L}_{t}\left[v_{x x x}-(u v)_{x}\right] \\
\therefore u(x, s)=\frac{x}{s}+\frac{1}{s^{2}}-\frac{-2}{s^{3}}+\frac{2 x}{s^{2}}-\frac{1}{s} \mathfrak{L}_{t}\left[\left(v^{2}\right)_{x}\right]
\end{gathered}
$$




$$
v(x, s)=-\frac{x}{s}+\frac{1}{s^{2}}-\frac{2 x}{s^{2}}+\frac{1}{s} \mathfrak{L}_{t}\left[v_{x x x}-(u v)_{x}\right]
$$

Taking inverse Laplace transform

$$
\begin{gathered}
u(x, t)=x+t-t^{2}+2 x t-\mathfrak{L}_{t}^{-1}\left\{\frac{1}{s} \mathfrak{L}_{t}\left[\left(v^{2}\right)_{x}\right]\right\} \\
v(x, t)=-x+t-2 x t+\mathfrak{L}_{t}^{-1}\left\{\frac{1}{s} \mathfrak{L}_{t}\left[v_{x x x}-(u v)_{x}\right]\right\}
\end{gathered}
$$

Consider solution of equation (22) is in the series form

$$
\begin{aligned}
& u(x, t)=\sum_{n=0}^{\infty} u_{n}(x, t) \quad \text { and } \quad v(x, t)=\sum_{n=0}^{\infty} v_{n}(x, t) \\
& \therefore \sum_{n=0}^{\infty} u_{n}(x, t)=x+t-t^{2}+2 x t-\mathfrak{L}_{t}^{-1}\left\{\frac{1}{s} \mathfrak{L}_{t}\left[\sum_{n=0}^{\infty} \mathcal{E}_{n}\right]\right\} \\
& \sum_{n=0}^{\infty} v_{n}(x, t)=-x+t-2 x t+\mathfrak{L}_{t}^{-1}\left\{\frac{1}{s} \mathfrak{L}_{t}\left[v_{n_{x x x}}-\sum_{n=0}^{\infty} \mathcal{C}_{n}\right]\right\}
\end{aligned}
$$

Take

$$
u_{0}(x, t)=x \quad \text { and } \quad v_{0}(x, t)=-x
$$

$$
\begin{aligned}
& u_{1}(x, t)=t-t^{2}+2 x t-\mathfrak{L}_{t}^{-1}\left\{\frac{1}{s} \mathfrak{L}_{t}\left[\mathcal{E}_{0}\right]\right\} \\
& v_{1}(x, t)=t-2 x t+\mathfrak{L}_{t}^{-1}\left\{\frac{1}{s} \mathfrak{L}_{t}\left[v_{0_{x x x}}-\sum_{n=0}^{\infty} \mathcal{C}_{0}\right]\right\} \\
& u_{n+1}(x, t)=-\mathfrak{L}_{t}^{-1}\left\{\frac{1}{s} \mathfrak{L}_{t}\left[\sum_{n=0}^{\infty} \mathcal{E}_{n}\right]\right\}, \quad n=1,2,3, \ldots \\
& v_{1}(x, t)=t-2 x t+\mathfrak{L}_{t}^{-1}\left\{\frac{1}{s} \mathfrak{L}_{t}\left[v_{0_{x x x}}-\sum_{n=0}^{\infty} \mathcal{C}_{0}\right]\right\} \\
& v_{n+1}(x, t)=\mathfrak{L}_{t}^{-1}\left\{\frac{1}{s} \mathfrak{L}_{t}\left[v_{n_{x x x}}-\sum_{n=0}^{\infty} \mathcal{C}_{n}\right]\right\}, \quad n=1,2,3, \ldots \\
& \therefore u_{1}(x, t)=t-t^{2}+2 x t-\mathfrak{Q}_{t}^{-1}\left\{\frac{1}{s} \mathfrak{Q}_{t}[2 x]\right\} \\
& u_{1}(x, t)=t-t^{2}+2 x t-2 x t=t-t^{2} \\
& \therefore v_{1}(x, t)=t-2 x t+\mathfrak{Q}_{t}^{-1}\left\{\frac{1}{s} \mathfrak{Q}_{t}[2 x]\right\} \\
& v_{1}(x, t)=t-2 x t+2 x t=t \\
& u_{2}(x, t)=-\mathfrak{L}_{t}^{-1}\left\{\frac{1}{s} \mathfrak{L}_{t}\left[\mathcal{E}_{1}\right]\right\}=-\mathfrak{L}_{t}^{-1}\left\{\frac{1}{s} \mathfrak{L}_{t}[-2 t]\right\}=t^{2} \\
& v_{2}(x, t)=\mathfrak{L}_{t}^{-1}\left\{\frac{1}{s} \mathfrak{L}_{t}\left[v_{1 x x x}-\mathcal{C}_{1}\right]\right\}=\mathfrak{L}_{t}^{-1}\left\{\frac{1}{s} \mathfrak{L}_{t}\left[-t^{2}\right]\right\}=-\frac{t^{3}}{3} \\
& u_{3}(x, t)=-\mathfrak{Q}_{t}^{-1}\left\{\frac{1}{s} \mathfrak{L}_{t}\left[\mathcal{E}_{2}\right]\right\}=-\mathfrak{L}_{t}^{-1}\left\{\frac{1}{s} \mathfrak{L}_{t}\left[\frac{2 t^{3}}{3}\right]\right\}=-\frac{t^{4}}{6} \\
& v_{3}(x, t)=\mathfrak{L}_{t}^{-1}\left\{\frac{1}{s} \mathfrak{Q}_{t}\left[v_{2 x x x}-\mathcal{C}_{2}\right]\right\}=\mathfrak{Q}_{t}^{-1}\left\{\frac{1}{s} \mathfrak{L}_{t}\left[t^{2}+\frac{t^{3}}{3}\right]\right\}=\frac{t^{3}}{3}+\frac{t^{4}}{12}
\end{aligned}
$$

Similarly, $\quad u_{4}(x, t)=\frac{t^{4}}{6}+\frac{t^{5}}{30} \quad$ and $v_{4}(x, t)=-\frac{t^{4}}{12}-\frac{t^{5}}{30}$ 
ISSN 2250-3153

Hence solution of equation (22) is

$$
\begin{array}{cc}
u(x, t)=x+t & u(x, t)=x+t-t^{2}+t^{2}-\frac{t^{4}}{6}+\frac{t^{4}}{6}+\frac{t^{5}}{30}-\cdots \\
v(x, t)=t-x & v(x, t)=t-\frac{t^{3}}{3}+\frac{t^{3}}{3}+\frac{t^{4}}{12}-\frac{t^{4}}{12}-\frac{t^{5}}{30} \\
&
\end{array}
$$

In this case noise terms are occurred so by cancelling the terms with opposite signs we get exact solution of equation (22).

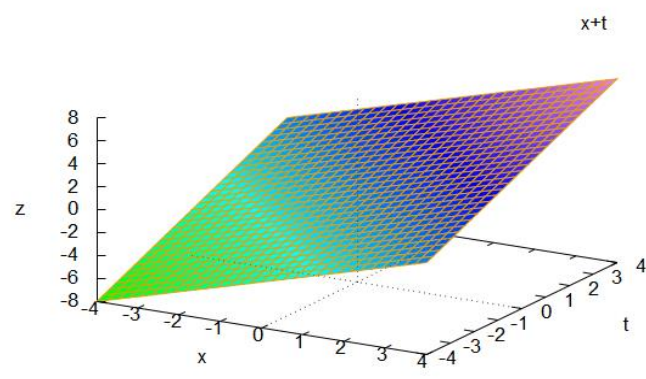

(a) $\boldsymbol{u}(\boldsymbol{x}, \boldsymbol{t})=\boldsymbol{x}+\boldsymbol{t}$

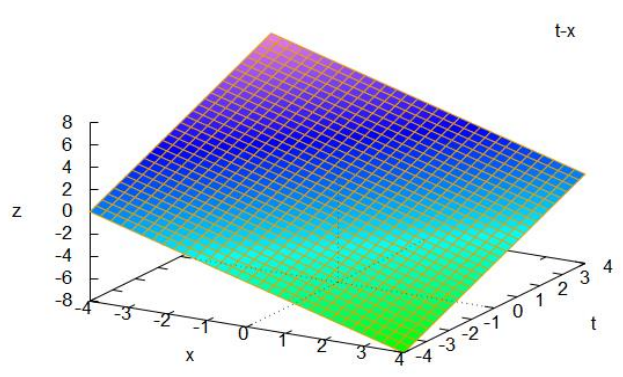

(b) $v(x, t)=t-x$

Figure 3: Graph of solution of Drinfield Sokolov system for $-\mathbf{4} \leq \boldsymbol{x}, \boldsymbol{t} \leq \mathbf{4}$

\section{Conclusion:}

LDM and modified LDM have been successively employed to find approximate analytical solution of DS system, coupled Burger's equation and Cauchy problem. Result obtained by these methods is investigated by numerical table (1) and (2) for coupled Burger's equation and Cauchy problem. In case of DS system due to existence of noise terms we get exact solution. Obtained result shows very good endorsement with exact solution. These methods reduce the volume of calculation as well as avoid the tedious calculations and provide good result in very few iterations. Therefore, LDM mLDM are strong mathematical tools to solve system of NLPDEs and can be used to solve linear, nonlinear partial differential equations which are widely used in different fields.

\section{References:}

[1] F. Zhaosheng, The first integral method to study the Burgers-Korteweg-de Vries equation, J. of Physics A. Mathematical and general, volume 35, issue 2 (2002), 343-349.

[2] R. Jebari, I. Ghanmi, A. Boukricha, Adomian decomposition mrthod for solving nonlinear heat equation with exponential nonlinearity, I. J. of Math. Analysis, volume 7, issue 15(2013), 725-734.

[3] B. Zheng, Exp-function method for solving fractional partial differential equations, Hindawi publishing corporation the sci. world J. volume 2013, article id 465723, 8 pages.

[4] D. J. Evans, K. Raslan, The tanh function method for solving some important nonolinear partial differential equations, in. J. of comp. math., volume 82, issue 7(2005), 897-905.

[5] A. Bekir, A. Cevikel, The tanh-coth method combined with the Riccati equation for solving nonlinear coupled equation in mathematical physics, J. of king saud university-sci. volume 23, issue 2 (2011), 127-132.

[6] J. Biazar, M. Eslami, A new homotopy perturbation method for solving system of partial differential equations, Comp. and math with appl., volume 62, issue 1 (2011), 225-234.

[7] M. Elsayed, A. Khaled, The homogeneous balance method and its applications for finding the exact solutions for nonlinear evolution equations, Italian J. of pure and appl. Math. Volume 33 (2014) 307-318.

[8] S. Handibag, Laplace decomposition method for the system of non linear PDEs, open access library journal, volume 6, (2019).

[9] M. Hussain, M. Khan, Modified laplace decomposition method, Appl. Math. Sci. volume 4, issue 36(2010), $1769-1783$. 
[10] M. Alquran, Applying differential transform method to nonlinear partial differential equations, An international J. ,volume 7, issue 1(2012) 155-163.

[11] M. Inc, E. Fendoglu, H. Triki and A. Biswas, Compactons and topological solitons of the Drinfield sokolov system, nonlinear analysis modelling and control, volume 19, issue 2 (2014), 209-224.

[12] S. Khuri, A Laplace decomposition algorithm applied to a class of nonlinear differential equations, Hindawi publishing corp. J. of appl. Math., volume 1, issue 4 (2001), 141-155.

[13] S. Esipov, Coupled Burgers equation, A model of polydispersive sedimentation, Phy. Rev. E. volume 52, issue 4(1995), 3711-3718.

[14] R. Abazari, A. Borhanifar, Numerical study of the soliton of the Burger and coupled Burgers equation by differential transform method, comp. and math. with appl., volume 59(2010), 2711-2722.

[15] A. M. Wazwaz, Exact and explicit travelling wave solutions for the non linear Drinfield sokolov system, communication in non linear sci. and numerical simu., volume 11 (2006), 311-325.

[16] F. Zhang, J. ming Qi, W, Yuan, Further results about travelling wave exact solutions of the Drinfield Sokolov equation, J. of appl. Math. volume 2013, article Id 523732, 6 pages.

[17] H. Cai, C. Pan, Z. Liu, some interesting bifurcation of nonlinear waves for the generalized Drinfield sokolov system, Hindawi publi. Corp. abstract and applied analysis, volume 2014, artical id 189486, 20 pages.

[18] J. Wang, A. List of 1+1 dimensional integrable equations and their properties, J. of nonlinear math. Phy., volume 9(2002), 213-233.

[19] A. Wazwaz, A new algorithm for calculating adomian polynomials for nonlinear operators. App. Math. And comp. , volume 111, issue 1 (2000) 33-51.

[20] N. Sweilama, M. Khader, Exact solutions of some coupled nonlinear partial differential equations using the homotopy perturbation method, comp. and math. with appl, volume 58 (2009), 2134-2141.

[21] A. Emad, K. Dawoud, M. marashdeh, Numeric-analytic solution of mixed-type system of balance law, appl. math. and computation, volume 265 (2015), 133-143.

[22] E. Eladdad, E. Tarif, On the coupling of the homotopy perturbation method and new integral transform for solving system of partial differential equations, Hindawi advances in Math. Phy. ,volume 2019, article Id 5658309, 7 pages. 